\title{
On the performance of the Gaia Auto-collimating Flat Mirror Assembly - Could it be even better?
}

\author{
A. Bos ${ }^{a, b}$, G. Witvoet $^{b}$, H.P. Pereboom ${ }^{b}$, M. Steinbuch ${ }^{a}$ \\ ${ }^{a}$ Eindhoven University of Technology, Control System Technology, Department of Mechanical \\ Engineering, Gem-N 0.68, P.O. Box 513, 5600 MB Eindhoven, The Netherlands; \\ ${ }^{b}$ TNO, Opto-mechatronics Department, Stieltjesweg 1, 2628 CK Delft, The Netherlands
}

\begin{abstract}
The Auto-collimating Flat Mirror Assembly (AFMA) simulated the rotation of the Gaia satellite around its vertical axis. As such, it was one of the most important ground support equipment tools to test the payload performance of the Gaia mission. Although the performance of TNO's design during commissioning was excellent, one of the challenges of the AFMA design and realisation has proven to be the prediction of the dynamic behaviour of AFMA and the accurate prediction of its performance. Therefore, the dynamic behaviour of Gaia AFMA has recently been re-analysed. Dynamical points of attention are shown, and possible design improvements have been derived.
\end{abstract}

Keywords: Space, Gaia mission, Gaia satellite, Optical Ground Support Equipment, Dynamic Analysis, FEA, EMA

\section{INTRODUCTION}

To test the performance of Gaia's payload module (Fig. 1), the Auto-collimating Flat Mirror Assembly (AFMA) was required to simulate the rotation of the Gaia satellite around its vertical axis. TNO designed two AFMA's. TNO was also involved in the design of the Basic Angle Monitoring ${ }^{1}$ (BAM) subsystem and Wave Front Sensor ${ }^{2}$ (WFS).

Although AFMA performed within specification, some of the dynamical behaviour could not be fully explained during the project (2008). Therefore, the dynamic behaviour of Gaia AFMA has recently been re-analysed, which is described in this paper. After an introduction to the Gaia space mission, the performance of Gaia AFMA is shown in Section 1. The mechanical design is briefly described in Section 2. Section 3 shows the dynamic analysis. Finally, the lessons learned are given in Section 5, before ending with a discussion.

\subsection{Gaia space mission}

Gaia $^{3}$ is a global space astrometry ESA mission, launched in December 2013. Gaia observes each target star about 100 times over a five-year period, measuring its distance, movement, and change in brightness. Gaia is building a 3D map of about one billion stars throughout our Galaxy with an astrometric accuracy of better than 24 parcsec at magnitude 15 (4000 times fainter than the naked eye limit), which is comparable to measuring the diameter of a human hair at a distance of $1000 \mathrm{~km} .{ }^{4}$

Further author information: (Send correspondence to A. Bos.)

A. Bos: E-mail: a.bos@tue.nl, Telephone: +31 402474580

Space Telescopes and Instrumentation 2016: Optical, Infrared, and Millimeter Wave, edited by

Howard A. MacEwen, Giovanni G. Fazio, Makenzie Lystrup, Proc. of SPIE Vol. 9904,

99044F · ( ) 2016 SPIE · CCC code: 0277-786X/16/\$18 · doi: 10.1117/12.2233169

Proc. of SPIE Vol. 9904 99044F-1 


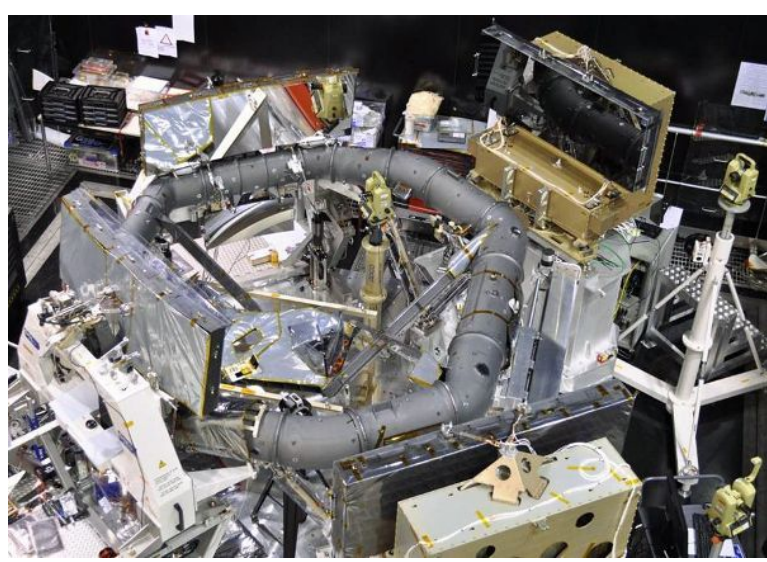

Figure 1: Gaia payload module and two realised AFMAs (one in the top right corner, one at the bottom). Courtesy of Airbus Defence and Space.

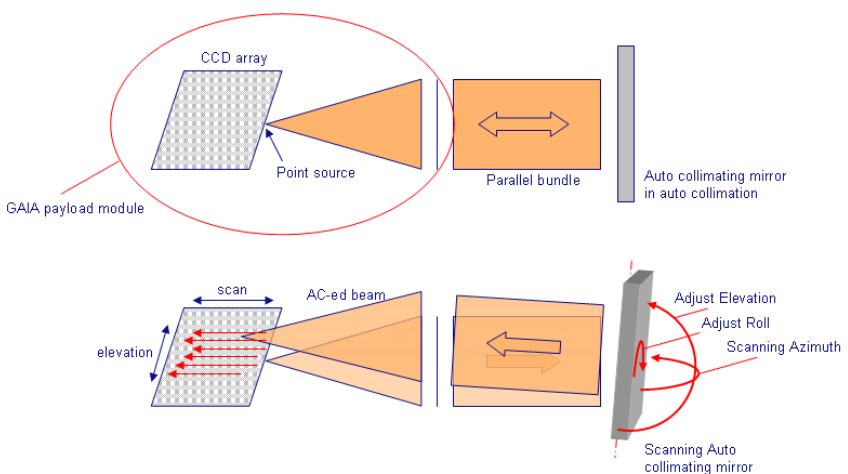

Figure 2: Gaia AFMA scan principle. A laser point source, positioned at the CCD-array, is retro reflected onto this array via a scanning auto-collimating mirror positioned opposite Gaia's telescope mirror.

\subsection{Gaia Auto-collimating Flat Mirror Assembly}

A laser point source, positioned at a CCD-array, is retro reflected onto the CCD-array via a scanning autocollimating mirror positioned opposite to Gaia's telescope mirror (Fig. 2). The AFMA mechanism is used to hold and actuate the Auto Collimating Flat mirror (AC-Flat) made out of Silicon Carbide (SiC) and weighing $90 \mathrm{~kg}$. By altering elevation and roll orientation of the scan axis the projected spot is adjusted to the required position on the CCD-panel. The reflected source is scanned on the Gaia CCD-panel comparable to the actual mission-stars in orbit.

The scanning performance around the vertical axis (azimuth), with a 20 mrad range and nrad-stability requirement, was most critical. Only this azimuth scanning-performance is considered in this paper.

\subsection{Main requirements}

On the satellite, the image of a star has a linear movement on the Focal Plane Array due to the spin motion of the satellite. The trace of the star is along the horizontal axis. The mirror had to rotate $\pm 10 \mathrm{mrad}$ around the vertical $z$ (Azimuth) axis, located in the mirror surface. The rotational velocity is $145.444 \mathrm{\mu rad} / \mathrm{s}$ with a required mean velocity stability of $3.2 \mathrm{nrad}$ over $4.4 \mathrm{~s}$. During this period the actual velocity should not differ more than $14 \mathrm{nrad} / \mathrm{s}$ from the mean velocity. The mirror axis also had to rotate around $y$-direction (elevation) in order to address the whole CCD-array. A fine adjustment around the $x$-axis (roll) was necessary to adjust the trace of the star along the scan direction. As the tests took place in a vacuum chamber, the mechanism had to be vacuum compatible and the motions had to be motorised. The $\mathrm{SiC}$ mirror and the Invar bipods were designed by Airbus Defence and Space (former Astrium). To be able to cool down the mirror separately from the AFMA framework, it was necessary to have free space around the mirror, which was required for thermal shielding. Therefore, the allowed volume for the mechanism was L-shaped.

\subsection{Performance}

Meeting the required nrad stability has been one of the challenges in the project. This was partly caused by ground vibrations and the dynamic interaction with the back structure, mostly low-frequent in nature, which were contributing most to the steady-state position error. To suppress these low-frequent contributions, sufficient disturbance suppression at these frequencies is required. This results in requirements for the controller sensitivity at these frequencies and thus the controller bandwidth (BW); the controller sensitivity (below the controller bandwidth, which is defined as the $0 \mathrm{~dB}$ crossing in the open-loop transfer function) automatically decreases when the controller bandwidth of the system is increased. However, achieving the required bandwidth was challenging, mainly because of some interesting discrepancies between the predicted and measured open loop transfer functions. In Fig. 3, a comparison with a 3D FEA model and a measurement from 2008 is shown.

In the measurement, Gaia AFMA is supported on an optical isolation table. Note that the difference between the 


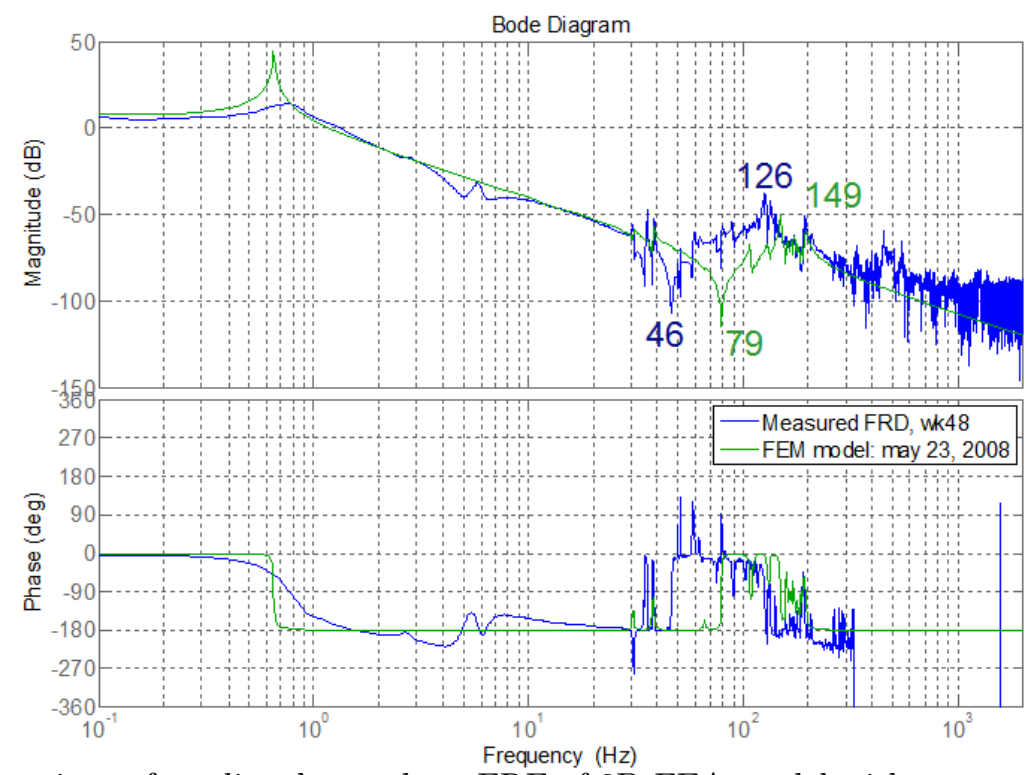

Figure 3: Comparison of predicted open loop FRF of 3D FEA model with a measurement (2008).

3D FEA model and the measurement (Fig. 3) is predominantly a lower decoupling resonance and the preceding anti-resonance at $79 \mathrm{~Hz}$ and $46 \mathrm{~Hz}$, which decreases the obtainable controller BW. This is probably caused by an overestimation of a connection stiffness. Furthermore, additional dynamics pops-up around the 30-60 Hz region. Especially the non-collocated resonance around $30 \mathrm{~Hz}$ (resulting in phase-lag instead of phase-lead) was never explicitly investigated and could not be explained during the project in 2008.

\section{MECHANICAL DESIGN}

In this section, the mechanical design of Gaia AFMA is described. First the concept is shown, before a design overview is given, where the details of the azimuth module is explained.

The AFMA azimuth module is a box-in-a-box design, with a rotating (inner) box connected to a fixed (outer) box (Fig. 4). A virtual axis of rotation for the scanning motion is positioned at the front surface of a SiC mirror, which is created by an elastic cross-hinge. This cross-hinge is assembled with four stiffened Ti6Al4V flexure hinges. The mirror is connected to the rotating (inner) box, and the cross-hinge connects this rotating box to the fixed (outer) box. The scanning motion is actuated by two modules connected to the outer box. Design improvements on these scanning modules have been developed.

\subsection{Concept}

AFMA is a stacked system that distinguishes the azimuth scan from the elevation- and roll-actuation. This approach ensures that cross talk motions are minimised during scanning. The allowed volume for the mechanism is L-shaped, resulting in the need for virtual axes of rotation. The Gaia AFMA mechanical layout is schematically illustrated in Fig. 4. The AC-Flat is directly mounted to a Mirror Frame (MF) with three Invar bipods. The Mirror Frame (MF) is connected via the elastic hinges (Virtual Pivot Mechanism, VPM) to the Base Frame (BF). Azimuth actuation of the Mirror Frame with AC-Flat is performed by two controlled voice coils (Scan Azimuth Module (SAM)). The Base Frame (BF) rests on the spherical sliding supports of the Support Frame (SF). To minimise friction and hysteresis, a weight compensation mechanism that lifts $87 \%$ of the weight is designed between the BF and the SF. Elevation and Roll are actuated by piezo motors.

\subsection{Detailed design overview}

In this subsection, the design of the azimuth module will be described in more detail. 


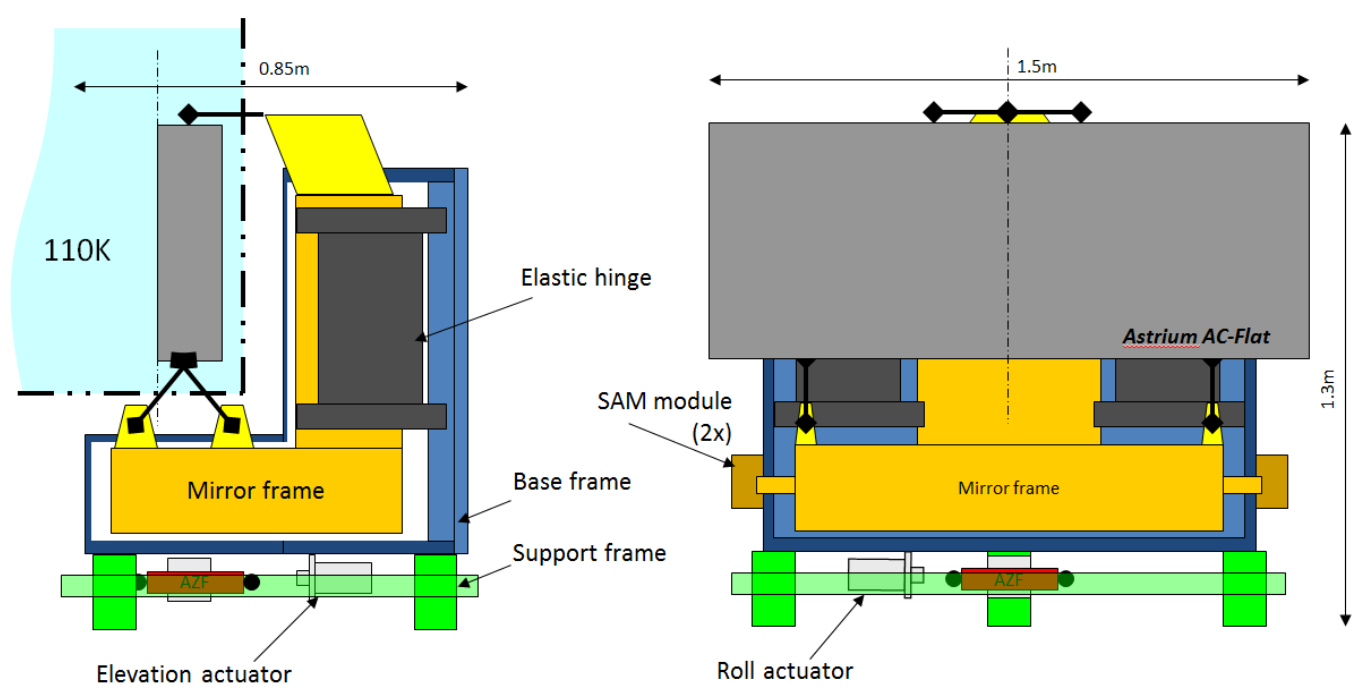

Figure 4: AFMA mechanical design concept. The AC-Flat is directly mounted to the mirror frame with three Invar bipods. The Mirror Frame is connected via the elastic hinges (Virtual Pivot Mechanism), to the Base Frame. Azimuth actuation of the Mirror Frame with AC-Flat is performed by two controlled voice coils (Scan Azimuth Module (SAM)). The Base Frame rests on the spherical sliding supports of the Support Frame. Elevation and Roll are actuated by piezo motors.

\subsubsection{Dummy Mirror (DM)}

The Dummy Mirror (DM) replaces the real $\mathrm{SiC}$ version and is made out of steel sheet metal. The DM weighs approximately $90 \mathrm{~kg}$. The Invar bipods are replaced with aluminium bipods with comparable properties. The DM and the bipods are visible in Fig. 5.

\subsubsection{Mirror Frame (MF)}

A design for a frame that has the highest possible stiffness in a given allocated volume can best be created by implementing closed boxes with the highest possible volume out of thin sheet metal to have the highest stiffness to mass ratio. For good thermal behaviour and low density, aluminium sheet metal of $3 \mathrm{~mm}$ has been chosen. The aluminium plates are connected to each other by butt-welding; this ensures stiff connections in combination with good dimensional stability. The MF is visible in Fig. 5 and weighs approximately $40 \mathrm{~kg}$.

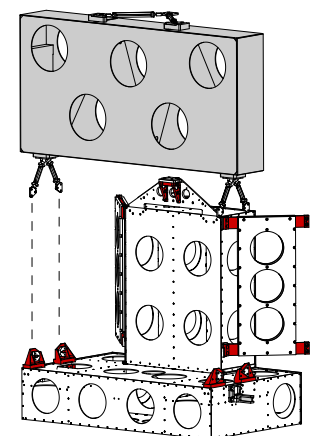

Figure 5: Dummy mirror, Mirror Frame and Virtual Pivot Mechanism. Grey: steel, white: aluminium, red: Ti6Al4V.
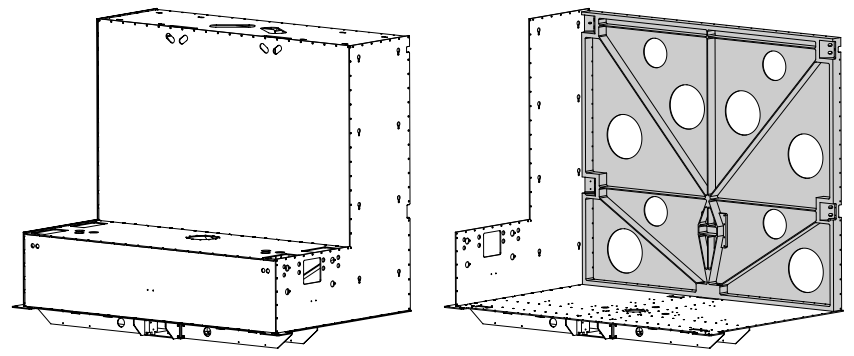

Figure 6: The Base Frame is constructed out of aluminium sheet metal. The back plate is stiffened. Right: $\mathrm{BF}$ where the stiffened back plate is coloured grey. 


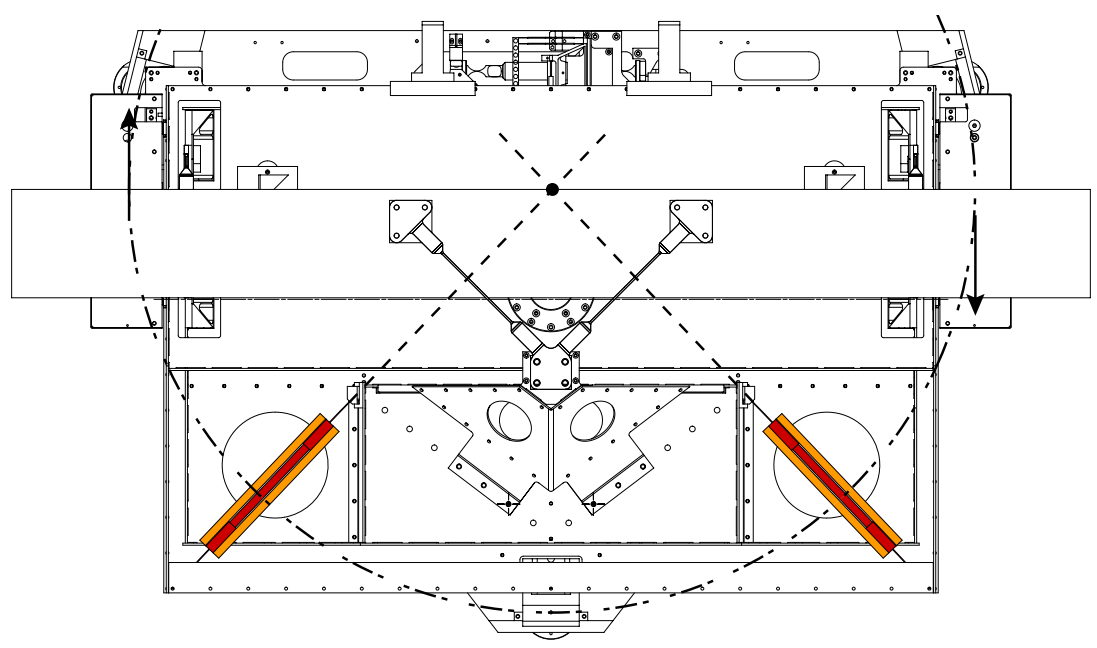

Figure 7: Top view of AFMA: the Virtual Pivot Mechanism (VPM) creates a virtual axis of rotation at the front of the mirror surface. Red: Ti6Al4V flexural hinge. Orange: Aluminium stiffened body. The mirror and the Mirror Frame (MF) are actuated at a radius of $0.6 \mathrm{~m}$.

\subsubsection{Virtual Pivot Mechanism (VPM)}

In order to prevent stick-slip and hysteresis effects, the design of the azimuth scan axis is based on cross-linked flexures. The virtual axis of rotation, which is created by the Virtual Pivot Mechanism (VPM), is positioned at the front surface of the $\mathrm{SiC}$ mirror. A top view of AFMA is shown in Fig. 7. The centre of rotation is located in the theoretical crossing of the two unbend flexures. Two sets of cross-linked pivots are mounted above each other with a stiff aluminium body in between. To actuate to the extreme azimuth orientation no more than 6 $\mathrm{Nm}$ is required. Each stiffened leafspring is made of Ti6Al4V and has a total length of $L=330 \mathrm{~mm}$, with a leaf spring length $l=41 \mathrm{~mm}$. The height is $H=50 \mathrm{~mm}$, with a thickness $t=1 \mathrm{~mm}$ (the stiffened parts are between 11 and $14 \mathrm{~mm}$ thick). The distance between the upper and lower stiffened leafspring is $550 \mathrm{~mm}$ (centre-centre). This results in a rotation stiffness of approximately $k_{z z}=425 \mathrm{Nm} / \mathrm{rad}$.

\subsubsection{Base Frame (BF)}

Just like the MF, the Base Frame (BF) is a butt-welded closed box constructed out of aluminium sheet metal (Fig. 6). The BF weighs approximately $50 \mathrm{~kg}$. The back plate of the $\mathrm{BF}$ is stiffened, because a moment is acting on the BF; the combined COG of the DM, MF and VPM is shifted with respect to the back plate.

\subsubsection{Scan Azimuth Module (SAM)}

The azimuth rotation is actuated by means of two voice coils, one at each side of the BF. The frame rotation is measured linearly with two optical position sensors with an interpolated resolution of $2 \mathrm{~nm}$. The azimuth angle is defined by averaging the sensor displacements and dividing by the radius (distance to centre of rotation). The sensors are co-located with their actuator as much as possible for the best possible loop shaping. A calibration of the sensors with an external interferometer system results in a look-up table for the controller in order to reach the required mean velocity on the internal sensors. One of the two Scan Azimuth Modules is depicted in Fig. 8. The frame of the SAM (white) is connected to the BF at four points (magenta). The SAM consists of a voice coil, where the magnet (dark grey) is connected to the MF (turquoise) via an intermediate body (green). To this intermediate body (green) a strut (red) is mounted. The strut is connected to a double parallelogram (grey) made out of aluminium, to which the linear encoder scale is mounted (not visible). The coil (orange) of the voice coil is connected to a reaction mass (RM) via an intermediate body (both yellow). This RM is guided via a single aluminium parallelogram (grey). 

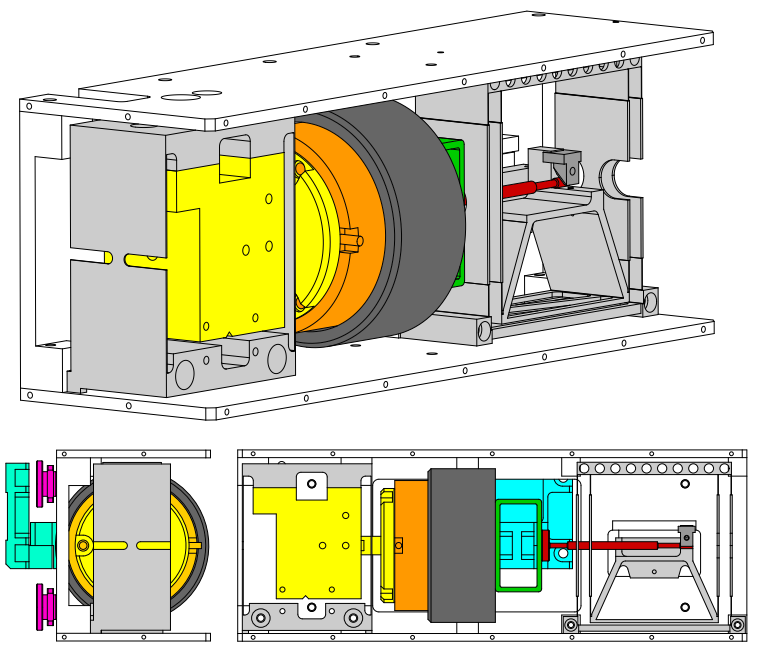

Figure 8: Scan Azimuth Module (SAM). The frame of the SAM (white) is connected to the BF at four points (magenta). The SAM consists of a voice coil, where the magnet (dark grey) is connected to the MF (turquoise) via an intermediate body (green). To this intermediate body (green) a strut (red) is mounted. The strut is connected to a double parallelogram (grey), to which the linear encoder scale is mounted (not visible). The coil (orange) of the voice coil is connected to a reaction mass via an intermediate body (both yellow). This reaction mass is guided via a single parallelogram (grey).

\section{DYNAMIC ANALYSIS}

Dynamic analyses have been carried out, starting with a comparison of a 1D dynamical model comprising of a mass-spring-damper model.

Furthermore, a finite element model (FEM) is constructed from a simplified 3D CAD model and a modal analysis has been performed. With the information of the modal analysis, and by defining the inputs and outputs, a modal state space description is obtained, being able to simulate a Frequency Response Function (FRF) to compare with the measurements. Also, an experimental modal analysis (EMA) has been performed and compared to the finite element analysis (FEA). The main dynamical points of attention are shown, and possible design improvements have been derived.

\subsection{D model}

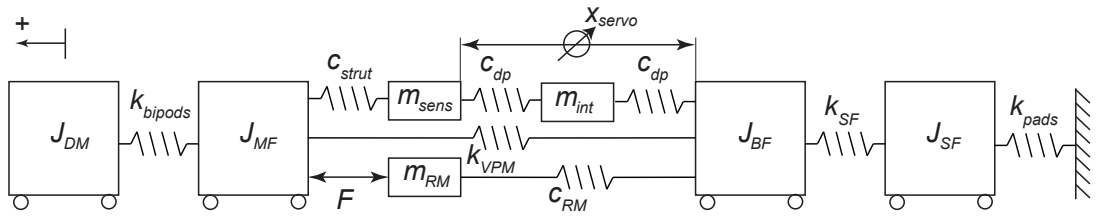

Figure 9: 1D mass-spring model. With $m_{i}$ the mass of a body, $J_{i}$ the mass moment of inertia, $C_{i}$ a translational stiffness and $K_{i}$ a rotation stiffness. The force acts between the MF and the RM. The output is measured between the sensor and the BF.

To describe and understand the basic behaviour, a 1D dynamical model is derived. The model, depicted in Fig. 9, describes the rigid body dynamics of the azimuth actuation of AFMA. The model consists of masses and inertias, seven in total, moving in line with each other. The masses and inertias are connected via linear springs and rotational springs, respectively. The force $F$ (input) acts between the Mirror Frame $J_{M F}$ and the Reaction Mass $m_{R M}$, the position readout (output) occurs between the linear encoder scale $m_{\text {sens }}$ and the Base Frame $J_{B F}$. 
Values of the parameters are based on simple calculations and the 3D CAD model. This results in an open loop frequency response as depicted in Fig. 10a, the modal damping is set to $\zeta=0.003$. The modal decomposition ${ }^{5}$ of the FRF is depicted in Fig. 10b, which shows the individual modal contributions to the frequency response. The sum of all the individual contributions results in the overall response (as depicted in Fig. 10a). The eigenfrequencies $f$ and the eigenvectors (mode shapes) $v$ of the model are shown in Table 1.

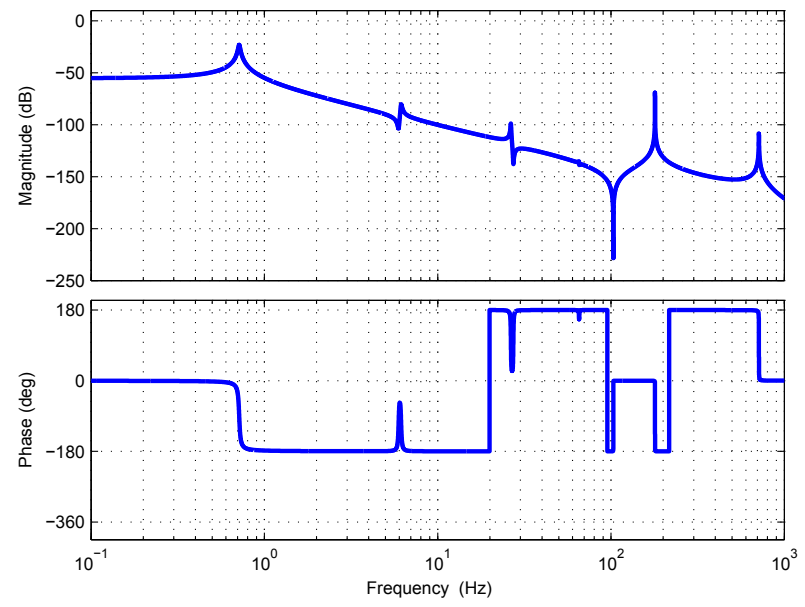

(a) Open loop FRF.

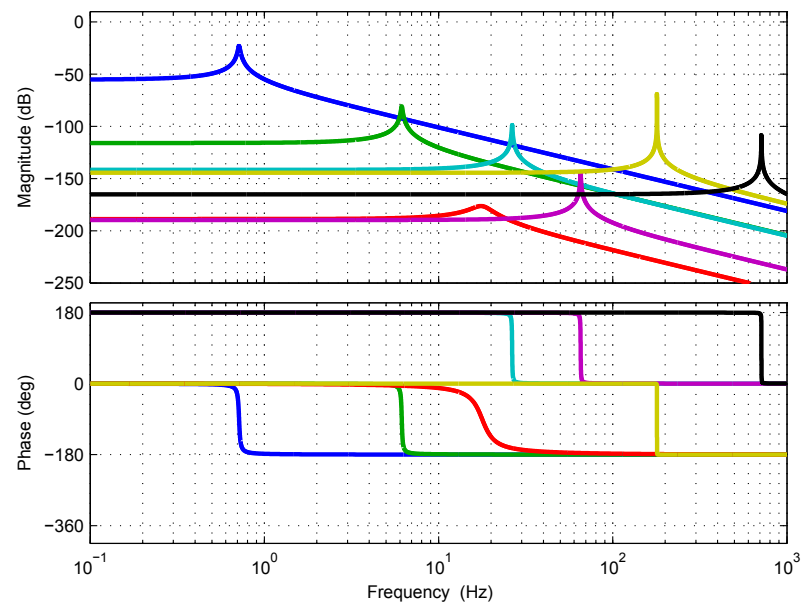

(b) Modal decomposition.

Figure 10: Frequency response of the 1D model.

Table 1: Eigenfequencies $f$ and eigenvectors $v$ of the 1D model

\begin{tabular}{|c|c|c|c|c|c|c|c|c|}
\hline & & Mode 1 & Mode 2 & Mode 3 & Mode 4 & Mode 5 & Mode 6 & Mode 7 \\
\hline Eigenfrequency $f \quad[\mathrm{~Hz}]$ & & 0.7 & 6.1 & 17.8 & 26.4 & 65.4 & 179.5 & 713.8 \\
\hline \multirow{7}{*}{$\begin{array}{ll}\text { Eigenvector } v & {[-]}\end{array}$} & $x_{D M}$ & 0.1876 & 0 & 0.0028 & -0.0001 & 0 & 0.1320 & -0.0005 \\
\hline & $x_{M F}$ & 0.1876 & 0 & 0.0027 & -0.0001 & 0 & -0.2667 & 0.0249 \\
\hline & $x_{R M}$ & 0.0001 & 0.7070 & 0 & -0.0115 & -0.0007 & 0 & 0 \\
\hline & $x_{B F}$ & 0.0001 & 0.0033 & 0 & 0.2004 & 0.0789 & 0 & 0 \\
\hline & $x_{S F}$ & 0 & 0.0011 & 0 & 0.0819 & -0.2081 & 0 & 0 \\
\hline & $x_{\text {sens }}$ & 0.1876 & 0 & 0.0017 & -0.0001 & 0 & -0.2848 & -4.4591 \\
\hline & $x_{i n t}$ & 0.0940 & 0 & -6.4543 & 0.0001 & 0 & 0.0014 & 0.0014 \\
\hline
\end{tabular}

The first mode at $0.7 \mathrm{~Hz}$ is the (designed) actuated mode, while the second mode at $6.1 \mathrm{~Hz}$ is basically the movement of the Reaction Mass (RM) on its guidance. Mode six at $179.5 \mathrm{~Hz}$ is the mode where the DM decouples from the MF.

In the remainder of the paper, a PIID+2nd order low-pass controller with the same order is applied to make it easy to compare systems. Furthermore, no notch filters are applied.

The stiffness between the SF and BF is relatively low, and these dynamics become BW-limiting (26.4 Hz); the phase-lag introduced at $26.4 \mathrm{~Hz}$ from $-180^{\circ}$ to $-360^{\circ}$ is BW-limiting. One of the options to increase the obtainable $\mathrm{BW}$ is to increase the stiffness of the SF and BF connections. Another option is to tune the reaction mass. To further examine the effect of the reaction mass, a 1D dynamical model without RM is derived (Fig. 11). This results in an open loop frequency response as depicted in Fig. 12a, the modal damping is set to $\zeta=0.003$ again. The modal decomposition of the FRF is depicted in Fig. 12b. The eigenfrequencies $f$ and the eigenvectors (mode shapes) $v$ of the model are shown in Table 2. 


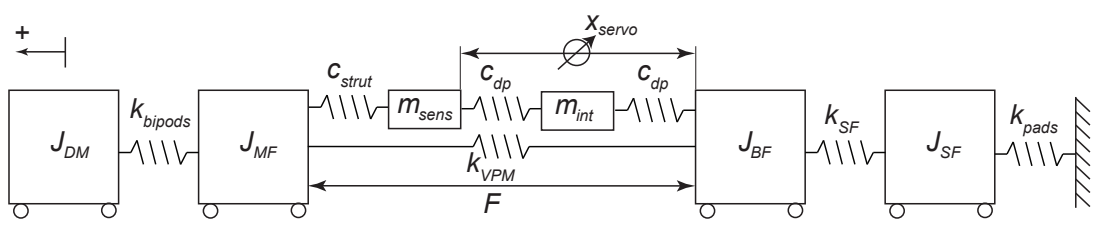

Figure 11: The 1D mass-spring model without RM. The force acts between the MF and the BF. The output remains unchanged, and is measured between the sensor and the BF.

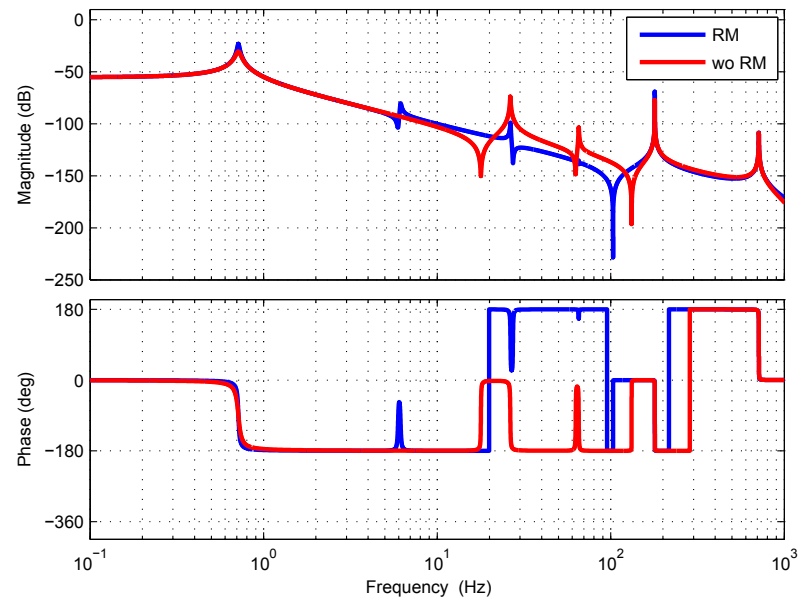

(a) Open loop FRF.

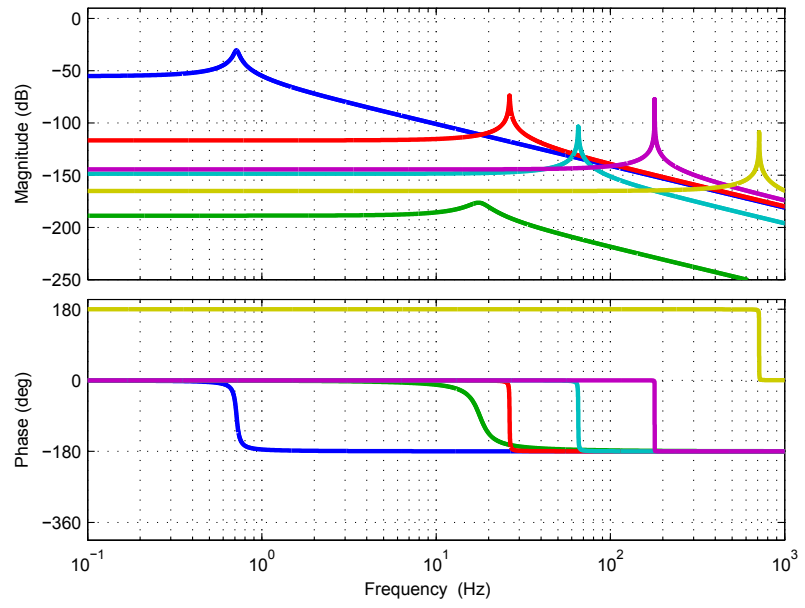

(b) Modal decomposition of FRF without RM.

Figure 12: Frequency response of the 1D model with and without RM.

Table 2: Eigenfequencies $f$ and eigenvectors $v$ of the 1D model without RM

\begin{tabular}{|c|c|c|c|c|c|c|c|c|}
\hline & & & Mode 1 & Mode 2 & Mode 3 & Mode 4 & Mode 5 & Mode 6 \\
\hline Eigenfrequency $f$ & {$[\mathrm{~Hz}]$} & & 0.7 & 17.8 & 26.4 & 65.4 & 179.5 & 713.8 \\
\hline \multirow[t]{6}{*}{ Eigenvector $v$} & {$[-]$} & $x_{D M}$ & 0.1876 & -0.0028 & 0.0001 & 0 & -0.1320 & -0.0005 \\
\hline & & $x_{M F}$ & 0.1876 & -0.0027 & 0.0001 & 0 & 0.2667 & 0.0249 \\
\hline & & $x_{B F}$ & 0.0001 & 0 & -0.2005 & -0.0788 & 0 & 0 \\
\hline & & $x_{S F}$ & 0 & 0 & -0.0818 & 0.2081 & 0 & 0 \\
\hline & & $x_{\text {sens }}$ & 0.1876 & -0.0017 & 0.0001 & 0 & 0.2848 & -4.4591 \\
\hline & & $x_{i n t}$ & 0.0940 & 6.4543 & -0.0001 & 0 & -0.0014 & 0.0014 \\
\hline
\end{tabular}

When we compare the eigenvectors and FRF of both models, we clearly see that the mode at $26.4 \mathrm{~Hz}$ in the FRF of the original model is less visible than in the model without the RM. This is easy to explain as in the original model, the force acts on the RM and the MF, while without the RM, the force acts on the BF and the MF. In the original model, the mode at $26.4 \mathrm{~Hz}$ is therefore less excited than in the model without RM; the RM acts as a mechanical low-pass filter. The same applies to the mode at $65.4 \mathrm{~Hz}$; in the FRF of the original model it is not visible. The attainable BW for the model with RM is in the order of $5 \mathrm{~Hz}$. For the model without RM, the same mode $(26.4 \mathrm{~Hz})$ is BW-limiting, but as the magnitude is higher, the maximum attainable BW is in the order of $2.5 \mathrm{~Hz}$, which increases the low-frequent disturbance suppression with a factor four $(12 \mathrm{~dB})$.

To further increase the effect of the RM, the resonance frequency of the second mode should be lower. This can be done by lowering the stiffness $c_{R M}$ or by increasing the mass of the RM $m_{R M}$. By decreasing the stiffness, the deflection of the RM would increase as the reaction force on the RM remains equal, which is considered unwanted. If the mass of the RM is increased from $2 \mathrm{~kg}$ to $5 \mathrm{~kg}$, this already results in a possible increase of the controller BW to $10 \mathrm{~Hz}$.

In conclusion, the stiffness of the $\mathrm{BF}$ and $\mathrm{SF}$ connection should ideally be larger. By implementing a reaction mass, these effects can be largely negated. However, the mass and stiffness combination of the reaction mass could be further optimised. Note that the 1D model only describes rigid body modes (i.e., infinitely stiff bodies), 
while the effect of flexible bodies is not taken into account yet.

\section{$3.23 \mathrm{D}$ model}

In this subsection, the dynamic analysis of a 3D model is carried out. Making use of a 3D CAD model, the flexible body modes and cross-coupling of the structure can be taken into account. A simplified CAD model forms the basis for a modal analysis. The eigenfrequencies and eigenmodes of the modal analysis are exported to MATLAB to generate a state space model. By choosing appropriate inputs and outputs, a frequency response is obtained to be compared with the measurements.

\subsubsection{FEA-based FRF}

To be abe to asses the quality of the 3D model, a comparison with measurements is made. For that, AFMA1 was installed in the TNO lab on its transport support. This transport support consists of a steel frame, connected to a steel transport frame mounted on three wire-rope isolators (Vertical stiffness $\approx 2 \cdot 4 \cdot 10^{5} \mathrm{~N} / \mathrm{m}$ ). Therefore, in the 3D CAD model, this support frame is modeled with two steel frames and connected with nine single-DOF spring-elements (Fig. 13). As boundary condition, the bottom of the transport support is fixed in all DOF. The first 100 eigenmodes are being calculated. Some modeshapes are visible in Fig. 14. From this modal analysis, it

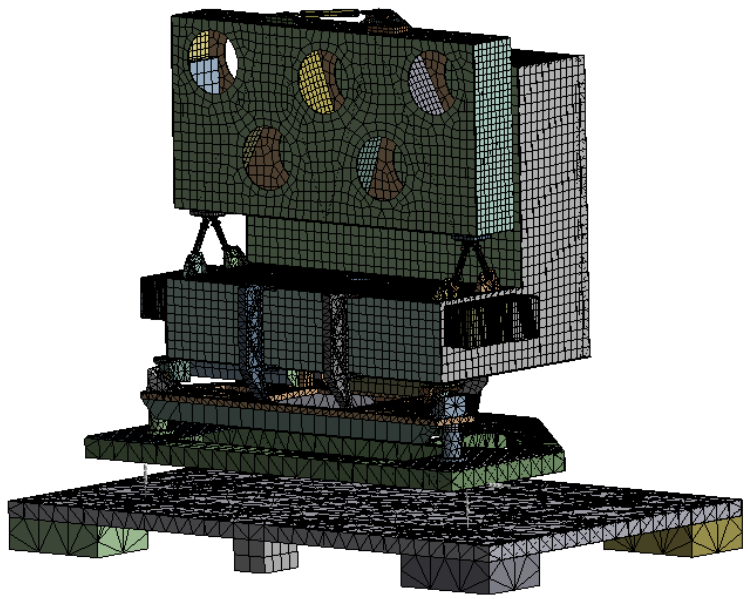

Figure 13: Simplified meshed FEM model of Gaia AFMA in ANSYS Workbench 15.0 - transport support included.

is possible to see that the stiffness of the BF and SF are not sufficient. The first flexible mode occurs around 27 $\mathrm{Hz}$ (this is a vertical movement, of which the second harmonic is shown at $42 \mathrm{~Hz}$ ), which is close to the estimated rigid body modes of the $1 \mathrm{D}$ dynamical models.

The mode around $5 \mathrm{~Hz}$ is a mode where the $\mathrm{RM}$ is moving on its guidance. The non-collocated resonance (phase-lag) around $17 \mathrm{~Hz}$ is a rotation mode of the support frame and $\mathrm{SF}$ and $\mathrm{BF}$ around $z$, while the MF and DM do not move. Moreover, the intermediate body of the double parallelogram is also resonating at this frequency. The mode around $17 \mathrm{~Hz}$ is an effect of the reaction path (reaction forces acting on the BF). Around $40 \mathrm{~Hz}$, a mode pops up which is a combination of torsion of the MF, vibration of the VPM bodies and rotation of the $\mathrm{BF}, \mathrm{SF}$ and transport frame on the wire rope isolators (around $z$ ). This is a flexible body mode.

In conclusion, it happens to be that the flexible body modes are close to the rigid body modes. For dynamic performance the stiffness of the intermediate bodies should ideally increase, as well as the stiffness of the connection between the bodies (to increase the rigid body eigenfrequencies). Moreover, some design improvements on the actuation module (SAM), where both the actuation and the measurement take place can be suggested (see Section 4). An interesting notion is that the missing 1:2 lever of the double parallelogram has a negligible effect on the FRF and the attainable BW. However, in reality, it is expected to have more influence, as one of the shortcomings of modal analysis is that it is a linear simulation, which does not take parasitic movement of a straightguide into account. 


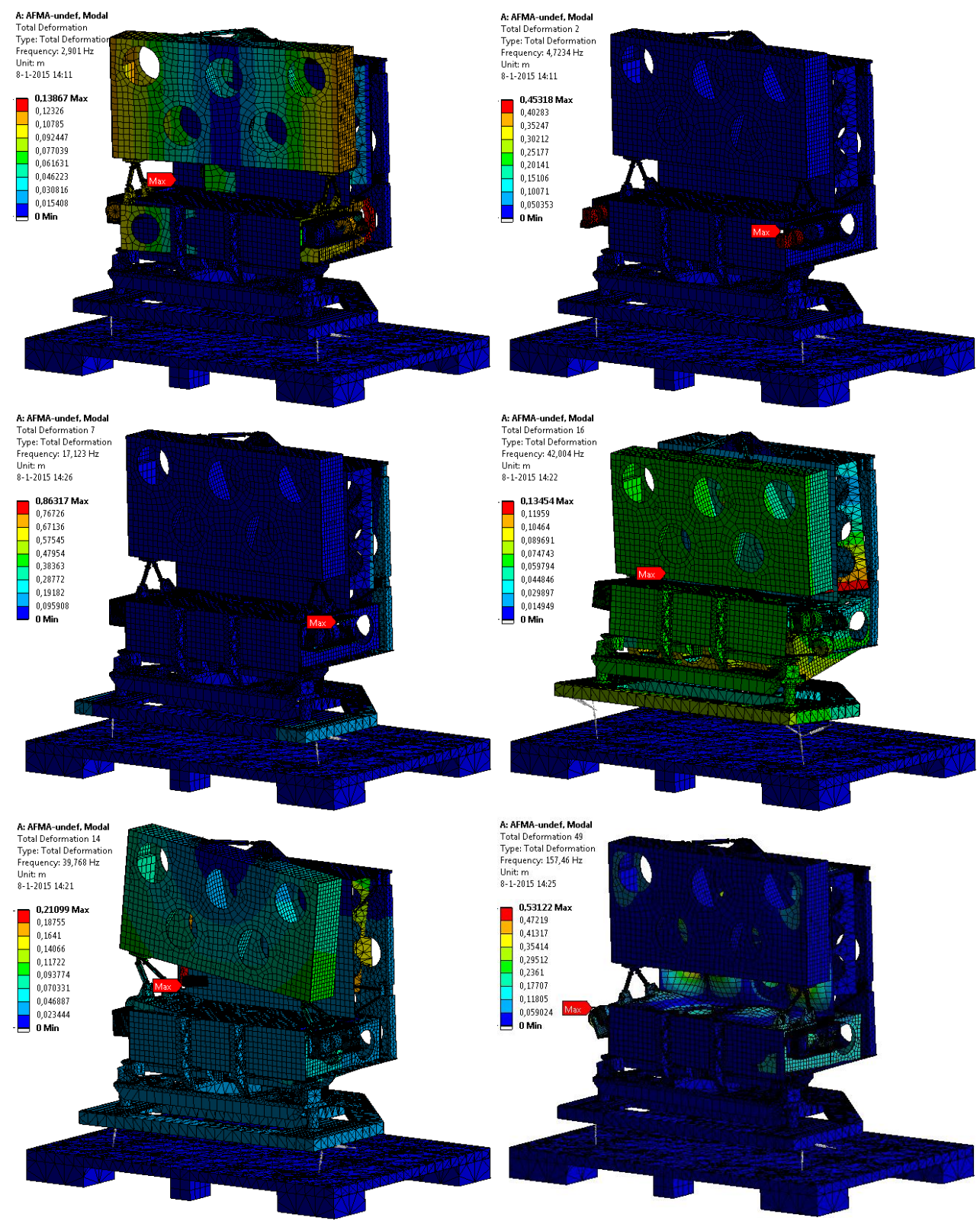

Figure 14: Modeshapes of Gaia AFMA with transport support structure.

\subsection{Measurements}

The measurements described in this subsection are performed on AFMA where the open loop FRF of the azimuth actuation is measured and compared with the effect of blocking the reaction masses.

\subsubsection{Measured FRF}

The open loop FRF measurement of the azimuth actuation is measured by injecting (filtered) noise at the input, getting a good signal-to-noise ratio. The effects of the reaction mass is investigated by blocking the RMs in the AFMA configuration. The comparison with the RM free and blocked are depicted in Fig. 15. The input is chosen as a force on the voice coil magnet (connected to the MF) and coil (connected to the BF), respectively. The output is chosen as the movement of the linear encoder scale (connected to the MF) with respect to the encoder readhead (connected to the BF). The forces and deflections are converted to a moment and rotation angle. 

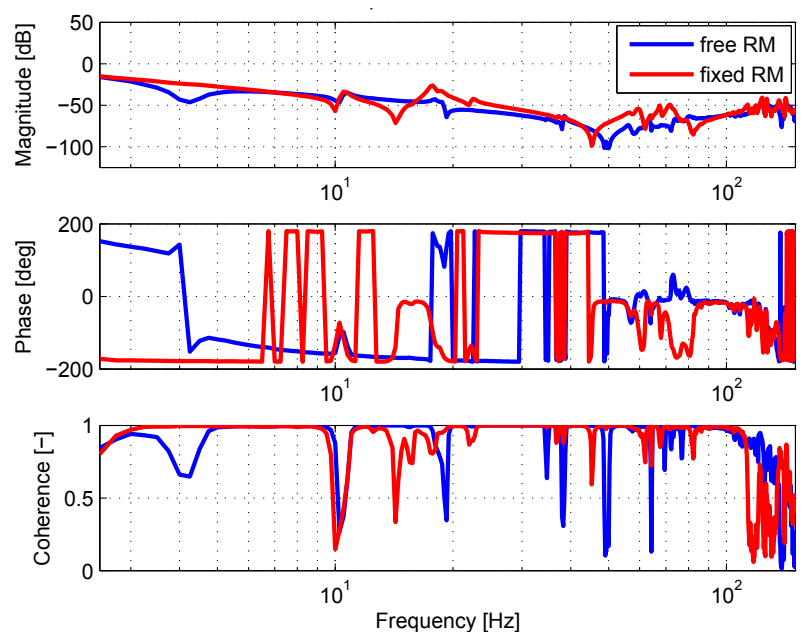

Figure 15: FRF measurement with free RM and blocked RM.
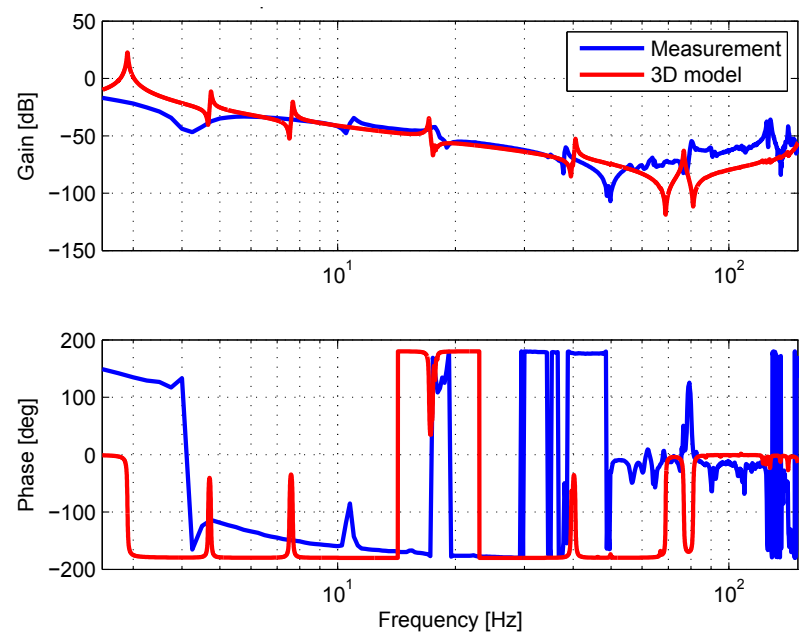

Figure 16: Comparison of the FRF of the 3D simplified model and the measurement in case of a free RM.

Around 4-5 Hz the anti-resonance and the highly damped resonance disappear without RM, although slightly shifted in frequency, this confirms that the $1 \mathrm{D}$ and 3D models have predicted this mode right. Furthermore, the first anti-resonance/resonance pair around $10 \mathrm{~Hz}$ does not change, which would be logical if it were a transport frame mode. After that, an anti-resonance/resonance pair with a large magnitude appears. This resonance is most likely the effect of the limited stiffness of the BF and SF and their connection, as already concluded in the 1D model. In fact, the 1D model describes the effect pretty well (see Fig. 12a).

\subsubsection{Comparison measurement and 3D model}

Finally, a comparison between the measurement and the 3D model on the transport frame is depicted in Fig. 16. Again, the modal damping is set to $\zeta=0.003$, and the same inputs and outputs are used.

The global shape of the FRF is about the same; the actuation mode (first resonance of the model) is not visible in the measurement, the second resonance, which is the RM-mode, is positioned at the correct frequency $(5 \mathrm{~Hz})$ but is highly damped in reality (probably due to eddy current damping of the voice-coil actuators). The third eigenfrequency, which is expected to be a transport frame mode, is estimated slightly too low. It is very well possible that the stiffness estimation of the wire rope isolators in certain directions is not correct, due to non-linear behaviour. The fourth (non-collocated) mode is again around the same frequency and the same applies to the fifth resonance. The major anti-resonance and decoupling resonance pop-up at a lower frequency in the measurement than estimated by the $3 \mathrm{D}$ model.

\subsubsection{Experimental modal analysis}

To correlate the the simulated modes with the measured modes, an Experimental Modal Analysis (EMA) is performed, using the roving hammer $\operatorname{method}^{6}$ and a Dewetron system. A total of five accelerometers are positioned on AFMA, and with hammer strikes the transfer functions are measured in the $x, y$ and $z$-direction at multiple positions on AFMA. The same strike-positions are re-used and the accelerometers are repositioned. One accelerometer always stays at the same position to extract the relative phase information.

The post-processing is done with FEMtools, where a comparison is made with the 3D FEM model. A transfer function is fitted on the measurements, a wire-model is generated and a Modal Assurance Criterion (MAC) analysis is performed. The post-processed data is compared to the FEM model, and comparable modes are extracted. This information can be found in Table 3. 
Table 3: Comparison mode shape pairs FEA and EMA

\begin{tabular}{ccccccc}
\hline \# & FEA & Hz & EMA & Hz & Diff. [\%] & MAC [\%] \\
\hline 1 & 3 & 4.7416 & 3 & 18.043 & -73.72 & 61.0 \\
2 & 4 & 6.7019 & 1 & 8.4352 & -20.55 & 98.6 \\
3 & 5 & 7.6729 & 2 & 10.547 & -27.25 & 96.2 \\
4 & 7 & 17.123 & 4 & 18.717 & -8.52 & 66.8 \\
5 & 8 & 17.410 & 5 & 22.271 & -21.83 & 61.1 \\
6 & 11 & 17.961 & 6 & 22.583 & -20.46 & 61.3 \\
7 & 14 & 39.768 & 8 & 36.982 & 7.53 & 91.4 \\
8 & 15 & 40.517 & 9 & 38.433 & 5.42 & 90.0 \\
9 & 16 & 42.004 & 10 & 40.295 & 4.24 & 95.9 \\
10 & 24 & 76.681 & 14 & 57.222 & 34.01 & 69.2 \\
11 & 25 & 84.842 & 20 & 65.918 & 28.71 & 64.4 \\
12 & 31 & 104.65 & 34 & 93.448 & 11.99 & 65.1 \\
\hline
\end{tabular}

According to the table, it looks like the fifth FEA mode (the third resonance in the 3D model FRF), indeed is a good simulation of the measured resonance around $11 \mathrm{~Hz}$. The same applies to the seventh mode of the FEA (fourth mode in the 3D model FRF) and the fourth EMA model around $19 \mathrm{~Hz}$. Although the simulated values are not spot-on with the measured values, this $3 \mathrm{D}$ model is very useful in the concept design process. This FEMtools comparison can be used for improvement of the 3D model by model-updating, but as our interests are in how to predict the behaviour in the design process, this will not clarify the actions one should take during modelling.

We were not able to show that the influence of the double parallelogram intermediate body is affecting the FRF in a negative way, because the double-parallelogram showed to be too delicate to put an accelerometer on. Furthermore, by measuring AFMA on its transport frame, eigenmodes shifted and effort was required to get a reasonable model of the support stiffness. The shifted eigenmodes and increased damping made it difficult to pinpoint the real problem dynamics using a technique like EMA in combination with FEMtools to correlate simulations to measurements.

Such measurements are better carried out on a heavy, stiff and vibration isolated table. This would minimise the cross-coupling of modes and also minimises damping.

\section{OTHER DESIGN RECOMMENDATIONS}

In this section some design improvements will be suggested, based on basic design principles. ${ }^{7,8}$

\subsubsection{Mirror Interfaces}

Moments are introduced by non-axial force paths in the connections from the mirror bipods to the MF. This introduces (bending) moments on the MF. A solution would be to replace the current interfaces with sandwich structures. The same applies to the the interface of the upper bipod. This should increase the connection stiffness of the DM to the MF.

\subsubsection{Scan Azimuth Module (SAM)}

Some design improvements can be given for the design of the SAM module (Fig. 8). For instance, the use of a single parallelogram $(l=90 \mathrm{~mm}, b=50 \mathrm{~mm}$ and $h=0.6 \mathrm{~mm})$ for the reaction mass $(2 \mathrm{~kg}$ moving mass) with a stroke of $\pm 4 \mathrm{~mm}$ results in a parasitic (vertical) movement of the reaction mass and the coil. This can result in (higher harmonic) components of the parallelogram being excited, and forces acting in directions perpendicular to the actuation direction. Moreover, the coil $(0.3 \mathrm{~kg})$ and the intermediate body $(0.1 \mathrm{~kg})$ are suspended by the single parallelogram, which will introduce a moment as the COG is shifted off-centre. Also, as the single parallelogram is a monolith, it is unnecessary to hinge one of the leaf springs, to increase the buckling force and decrease asymmetry in the guidance. Furthermore, the width of the parallelogram is relatively small compared to its height (a plate constrains $3 \mathrm{DOF}$, but by decreasing the width-to-height ratio it will start behaving like a strut more and more, which constrains only 1 DOF). Finally, the single parallelogram is mounted to the SAM 
frame on one side, which results in deformations.

The application of a double parallelogram $(l=92 \mathrm{~mm}, b=38 \mathrm{~mm}$ and $h=0.25 \mathrm{~mm})$ with a stiffened mid part $(l=79 \mathrm{~mm}, b=38 \mathrm{~mm}$ and $h=1.3 \mathrm{~mm})$ to act as a straight-guide for the linear encoder scale with a stroke of $\pm 4 \mathrm{~mm}$, is sound engineering practice. However, a 1:2 lever, which synchronises the movement of the main body and the intermediate body, is missing. This results in an undefined movement of the linear encoder scale and a low resonance frequency of the intermediate body. Again, as the double parallelogram is a monolith, it is unnecessary to hinge the stiffened part and the width is relatively small compared to its height.

The voice coil magnet is connected to the MF via an intermediate body. As the MF rotates, the voice coil magnet also rotates, which results in a change in the air-gap between the coil and the magnet. This may result in parasitic forces.

By applying these design improvements, it is expected that the resonance frequencies of the SAM module itself, and the components within, will increase. It is expected that some of the low frequent dynamics that are visible in the frequency response will move to higher frequencies, with a possible increase in controller bandwidth.

\section{LESSONS LEARNED}

The main lessons learned from back-engineering and re-analysing AFMA are given in this section.

Specific lessons regarding Gaia AFMA:

- moments are introduced by non-axial force paths, this also results in a decreased stiffness. By replacing the current interfaces with sandwich structures, this issue will be solved and an improvement in dynamic performance is expected.

- the SAM modules could be improved:

- by shifting the COG of the reaction mass assembly in the SAM towards the centre of the parallelogram, moments are prevented. Furthermore, as the single parallelogram is a monolith, it is unnecessary to hinge the leaf springs, which increases the buckling force and symmetry in the guidance. If using a double parallelogram to prevent parasitic (vertical) movement, a 1:2 lever should be added. Also, by mounting the reaction mass assembly from the bottom, using a sufficient stiff connection of the SAM to the BF, deflections are minimised. Finally, the width of the leafsprings is relatively small compared to its height (a plate constrains 3 DOF, but by decreasing the width-to-height ratio it will start behaving like a strut more and more, which constrains only 1 DOF).

- a 1:2 lever could be added to the stiffened double parallelogram that acts as a straight-guide for the linear encoder scale. In the present design, the movement of the encoder scale is undefined and the resonance frequency is very low. Again, as the double parallelogram is a monolith, it is unnecessary to hinge the stiffened part. The width-to-height ratio is also off in this design.

- from a dynamic point of view, the reaction mass could be tuned more optimally. By increasing the mass, the mechanical low-pass filtering effect could be increased.

- to improve the dynamic performance, the stiffness of the BF and SF connection should ideally increase. Furthermore, effort should be taken to increase the stiffness of the closed-box structures and to minimise mass, as the flexible body modes are only slightly higher than the rigid body modes.

General lessons:

- designing for high (dynamical) performance starts with light, stiff and statically-determined design. This often has a positive effect on the dynamic behaviour as well.

- the basic (rigid body) dynamics of a concept can be estimated with 1D mass-spring-damper models. However, estimations of the mass, inertia and (connection) stiffness needs to be in the right order to be of any use. If not, switching of eigenmodes can occur, which can alter the dynamical behaviour significantly. 
- FEA based on a 3D CAD model provides a good estimation of dynamic behaviour, especially information that can be found regarding flexible modes and cross-coupled modes. However, with large and complex systems, these simulations can become relatively expensive in view of simulation times, and depend largely on the boundary conditions. Simplified models with the main components will give the most time-efficient results. Furthermore, one needs to be aware of the limitations of FEA, being (amongst others): overestimated stiffness due to choice of boundary conditions and connections, and the fact that a modal analysis is based on linear simulation; second order influences cannot be found.

- using a reaction mass can be a viable approach to improve (dynamical) performance, as they work as a mechanical low-pass filter. Some general design rules for the application of a reaction mass have been derived. Two basic mass-spring-damper systems are defined:

1. a base connected to the fixed world with a spring, and a stage (without connection). The force acts between the base and the stage, and the position measurement takes place between the two bodies. When applying a reaction mass, the resonance frequency of the reaction mass should be chosen below the resonance frequency of the base, and ideally the difference in frequency should be as large as possible for the reaction mass to have the most effect. When looking at the frequency response, the application of the reaction mass will result in a loss of collocation at the resonance frequency of the base (phase-lag instead of phase-lead).

2. a base connected to the fixed world with a spring, and a stage connected to the base with a spring. The force acts between the base and the stage, and the position measurement is again done between the two bodies. When applying a reaction mass, the resonance frequency of the reaction mass should be chosen at least below the resonance frequency of the base, and ideally below the resonance frequency of the stage as well, because in the latter situation, collocation at the resonance frequency of the base will not be lost.

When the ideal resonance frequency range of the reaction mass is determined, this frequency can be generated by choosing a combination of a mass and stiffness. Increasing the mass or decreasing the stiffness will both decrease the resonance frequency of the reaction mass, but some practical concerns arise:

- increasing the mass should fit into the overall mass and volume budget of the system, and the (surrounding) supporting structures should have high enough a stiffness so their dynamics do not become limiting.

- decreasing the stiffness results in increasing amplitudes when the reaction forces stay the same. This influences the required actuator stroke and the required stroke of the reaction mass guidance system.

- if possible, do not use the transport frame of an instrument when doing EMA, as modeshapes can be highly influenced by the transport frame and it is difficult to model. Obviously, a transport frame is not designed for dynamic performance. Use a(n) (heavy and stiff) optical table or mount the instrument to the ground.

- exporting modeshapes from FEA to construct a state space model can give additional insight in possible problem dynamics, and the performance that can be attained by implementing a controller. However, in the concept design stage, modal analysis alone should give sufficient insight to improve the design during the first iterations.

- it is not guaranteed that FEA on a (simplified) CAD model will result in an accurate prediction of dynamical behaviour. Engineering knowledge is still required to be able to focus on the important parts of a complex design. 


\section{DISCUSSION}

By re-analysing Gaia AFMA from a dynamical point of view, some design improvements have been derived. The flexible body mode eigenfrequencies are close to the rigid body mode eigenfrequencies; an increase in both connection stiffness and stiffness of the closed-box structures would increase these frequencies. Furthermore, the effects of the reaction masses are described. However, we were unable to explain the non-collocated resonance around $30 \mathrm{~Hz}$ (resulting in phase-lag instead of phase-lead) that was found in old measurements. This is probably the result of measuring AFMA on a different support.

Predicting the (dynamic) performance of such a complex system has proven to be a non-trivial task. Using Experimental Modal Analysis and making use of FEMtools to correlate the FEA to the measurements can be useful to check the validity of your model.

To maximize (dynamic) performance the author suggests that during the design process, the focus must be on statically-determined, light and stiff design, optimising force paths and minimising moments. The use of 1D mass-spring-damper models can be highly effective to get a rough estimation of the dynamic behaviour of a certain concept. When progressing in the design, stiffness should be increased and mass minimised by performing static and modal FEA simulations, making use of stress distributions. Only after this step, converting the information of the modal analysis to a state space model, to simulate the dynamic behaviour and attainable performance, should be performed. Even then, one needs to be aware of the limitations of FEA.

\section{REFERENCES}

[1] Meijer, E., Nijenhuis, J., Vink, R., Kamphues, F., Gielesen, W., and Coatantiec, C., "Picometer metrology for the GAIA Mission," Proc. SPIE 7439 (2009).

[2] Vosteen, L., Draaisma, F., van Werkhoven, W., van Riel, L., M.H.Mol, and den Ouden, G., "Wavefront Sensor for the ESA-GAIA Mission," Proc. SPIE 7439 (2009).

[3] Mora, A., Biermann, M., Brown, A., Busonero, D., Carminati, L., Carrasco, J., Chassat, F., Erdmann, M., Gielesen, W., Jordi, C., Katz, D., Kohley, R., Lindegren, L., Loeffler, W., Marchal, O., Panuzzo, P., Seabroke, G., Sahlmann, J., Serpell, E., Serraller, I., van Leeuwen, F., van Reeven, W., van den Dool, T., and Vosteen, L., "Gaia on-board metrology: basic angle and best focus," Proc. SPIE 9143 (2014).

[4] www.esa.int, [Gaia overview] (last update: 23 April 2014).

[5] Rankers, A., [Machine Dynamics in Mechatronic Systems: an Engineering Approach (PhD thesis)] (1997).

[6] Ewins, D., [Modal Testing: Theory, Practice and Application] (2000).

[7] Schellekens, P., Rosielle, N., Vermeulen, H., Vermeulen, M., Wetzels, S., and Pril, W., "Design for Precision: Current Status and Trends," Annals of the CIRP 47/2 (1998).

[8] Rosielle, P., [Constructieprincipes, Lecture notes, Eindhoven University of Technolgy, in Dutch] (2007). 